\title{
Distribution of Earthquake Magnitude Levels in Bangladesh
}

\author{
Md. Habibur Rahman ${ }^{1} \&$ Md. Moyazzem Hossain ${ }^{1}$ \\ ${ }^{1}$ Department of Statistics, Jahangirnagar University, Bangladesh \\ Correspondence: Md. Habibur Rahman, Department of Statistics, Jahangirnagar University, Bangladesh. E-mail: \\ habib.drj@juniv.edu; habib.drj@gmail.com
}

Received: September 2, 2019

Accepted: September 23, 2019

Online Published: September 30, 2019

doi:10.5539/jgg.v11n3p15

URL: http://dx.doi.org/10.5539/jgg.v11n3p15

\begin{abstract}
Earthquakes are one of the main natural hazards which seriously make threats the life and property of human beings. Different probability distributions of the earthquake magnitude levels in Bangladesh are fitted. In terms of graphical assessment and goodness-of-fit criterion, the log-normal distribution is found to be the best fit probability distributions for the earthquake magnitude levels in Bangladesh among the probability distribution considered in this study. The average earthquake magnitude level found 4.67 (in Richter scale) for log-normal distribution and the approximately forty-six percent chance is predicted to take place earthquake magnitude in the interval four to five.
\end{abstract}

Keywords: Earthquake, Cumulative Distribution, Log-Normal, Anderson-Darling, AIC, BIC

\section{Introduction}

Bangladesh is the most earthquake venerable countries of the globe. Bangladesh is a South-Asian developing country which is used to struggle with a mixture of natural disasters and the earthquake is one of them. Bangladesh is in the right place to South Asia and lies between $20^{\circ} 34^{\prime} \mathrm{N}-26^{\circ} 38^{\prime} \mathrm{N}$ and $88^{\circ} 01^{\prime} \mathrm{E}-92^{\circ} 41^{\prime} \mathrm{E}$. The area of the country is approximately 148 thousand square kilometers with more than seven hundred and ten kilometers long coastlines and it covers about approximately eighty percent of the Bengal Basin. The land part is following a downward slope of one to two degree from north-west to south-east direction. Understanding the patterns in the frequency of natural events such as earthquakes is very important as it helps in the prediction of their future occurrence and hence provides better protection for human and different other lives. Distributions describing these events are known to be standard distributions unsuitable for modeling the frequency of such events. Bangladesh is frequently exposed to frequent damaging earthquakes for the reason that it is surrounded by high seismicity regions (Ansary, 2002). The fitting distribution to atmospheric data like earthquake magnitude is one of the very frequent tasks that choose probability distribution to model a random variable along with the estimates of the parameter(s) of the chosen distribution under efficient expertise and valid judgment, which generally necessitates iterative process of distribution choice, parameter estimation, and determining quality-of-fit assessment. It is thus finds interest in the field of meteorology (for any atmospheric parameter) to provide a good fit of atmospheric data which strongly depends on the fitting of the probability distribution.

The Birnbaum - Saunders distribution and its extreme value version have been applied to fit earthquake data and this application identified the extreme value Birnbaum-Saunders distribution as a better model than classic extreme value distributions for describing seismic events (Lillo et al., 2018). Nichols et al. (2018) finds the interdependency amongst earthquake magnitudes in Southern California and the results indicate that the positive correlation observed between larger earthquakes in the gCMT catalog and their aftershocks are also evident in the relationship between the magnitudes of earthquakes in the SCEDC data and their aftershocks. The artificial neural network (ANN) models are applied for mapping earthquake-triggered landslide susceptibility (Tian et al., 2019). Delignette-Muller et al. (2015) applied Akaike information criterion and Bayesian information criterion to develop a package to fit different distributions. The hierarchical clustering techniques and empirical distribution patterns are used to find similar regions based on the atmospheric data - precipitation of different region in Bangladesh (Rahman et al., 2018). Ara $(2013,2014)$ studied about the population distribution and its effect, and loss estimation procedures for earthquake risk assessment. The severity of earthquake-induced landslide events can be enumerated by the landslide-event magnitude, a metric derived from the frequency-size distribution of landslide inventories (Tanyaş et al., 2019). Roback et al. (2018) studied on the distribution, size and mobility of landslides caused by earthquake at Napal. Spatial distributions of earthquake of the northwest South Island, New Zealand induced 
landslides and hill slope are fitted (Parker et al., 2015). Mazzotti et al. (2005) estimate the rates of seismic moment and deformation in seismic zones of eastern Canada based of earthquake statistics from the Canadian earthquake hazard model and use a logic tree approach to derive median values and sixty six percent confidence intervals for models based on earthquake cluster distributions and geological and tectonic structures. Rahman et al. (2018) carried out the study to find out the probability of occurrence earthquake using the earthquake's data for the period of 1918 to 2016 and the parameters of weibull distribution and log-normal distribution, and the rate of exponential distribution are calculated for this data to fit the models. The Weibull distribution is the most significant distribution especially for reliability and maintainability analysis. Bhattacharya et al. (2009) presents analytical methods for estimating Weibull parameters, namely, shape parameter and scale paramete and the computational experiments on the presented methods The Earthquakes on a specified fault with magnitudes greater than a specified value have a statistical distribution of recurrence times. The exponential, Weibull and Rayleigh probability distribution functions are used to predict the earthquake occurrence in Japan (Ferraes, 2003). Abyani et al. (2019) proposed to make use of the sample geometric mean instead of the sample median to seismic reliability evaluation.

The Weibull (or stretched exponential) distribution, the log-normal distribution (Patel et al., 1976), and the Brownian passage-time (or inverse Gaussian) distribution (Matthews et al., 2002) are fitted for the earthquake waiting times and found that the synthetic data are well represented by Weibull distributions (Yakovlev et al., 2006). The Bayesian approach provides a mathematical tool for the treatment of uncertainties in modelling and data analysis (Bernardo and Smith, 1994). Pasari (2019) tried to forecast the earthquake using the Log-normal and Inverse Gaussian or Wald distribution of northeast India, northwest Himalaya, and western India. In the Bayesian view, probability is associated with a lack of knowledge and considered to measure the degree of belief that an event will occur (Sornette, 2000). Zöller et al. (2007) present a strategy for estimating the recurrence times between large earthquakes and associated seismic hazard on a given fault section. The deep artificial neural networks (ann) used as a tool for the analysis of the seismic data (Kislov et al., 2018).

Dhanya (2019) found that the majority of earthquake slip distributions follow truncated exponential or generalized Pareto distribution functions and the best-fitted distribution obtained by model fitting criteria like Akaike Information Criterion, mean squared error, correlation coefficient, and QQ plots. Roy (2014) fits two parameter Weibull distribution to find the probability of earthquake magnitude in Bangladesh and try to predict the earthquake at near feauture. The amount of energy released during an earthquake, which is computed from the amplitude of the seismic waves. A magnitude of 7.0 on the Richter scale indicates an extremely strong earthquake. Each whole number on the scale represents an increase of about 32 times more energy released than the previous whole number represents. Therefore, an earthquake measuring 6.0 is about 32 times more powerful than one measuring 5.0. Al Zaman and Munira (2017) have observed that most of the earthquake occurred in Bangladesh were not devastating but the occurrences of those small magnitude earthquakes have been increasing significantly under the area between $20.35^{\circ} \mathrm{N}$ to $26.75^{\circ} \mathrm{N}$ Latitude and $88.03^{\circ} \mathrm{E}$ to $92.75^{\circ} \mathrm{E}$ Longitude. Realistic assessment of seismic activities in Bangladesh may help out to reduce the risk from this catastrophic disaster, and the earthquake catalogues in this regard are the only sources as the most important products for studying seismological activities those can support to understand earthquake physics and let to learn seismotectonics, seismicity or seismic hazard of an area. The seismic status in Bangladesh has been explored using earthquake data recorded by the global network of USGS during 1980 to 2016 (Rahman et al., 2018). The Gumbel's asymptotic distribution of type three of the extreme value method is employed to estimate the earthquake hazard parameters in the Iranian Plateau (Mohammadi et al., 2016). Charpentier and Durand (2015) fit a Pareto distribution for earthquake magnitudes and waiting times are modeled using a Gamma or a Weibull distribution to estimate the probability of occurrence of several earthquakes within a year or a decade.

The main objectives of this research work are - to reveals the nature of earthquake magnitude, to fit and select the best-fitted probability distribution for earthquake magnitude in Bangladesh, to test the quality-of-fit of the probability distribution by the goodness-of-fit statistic, to judge the quality-of-fit of the probability distribution by the value of the log likelihood, Akaike information criterion (AIC), and Bayesian information criterion (BIC). In this research work especially develops and present following steps will be implemented: Collecting of Data of interest and exploratory data analysis is such as visualization, detection of outlier, trimming etc., the data must be taken into streamline, also as well as summarized, Reveals the nature of earthquake magnitude, Fit and select the best-fitted probability distribution for earthquake magnitude, test the quality-of-fit of the probability distribution by the goodness-of-fit statistic, Judge the quality-of-fit of the probability distribution by the value of the $\log$ likelihood, Akaike information criterion (AIC), and Bayesian information criterion (BIC). 
This study is organized into different sections including this section. The second section reviews the theory, methods and the methodology of different techniques related to fit the probability distribution. In this section, the basic concepts of the different probability distribution, cumulative distribution of probability distributions are discussed. Also in this section, the different goodness-of-fit tests for fitted probability distributions are talked about. The third section is center of attention on the computational issues and results of the fitted probability distributions. As well as the discussions about the results are presented in this segment. A summary with some concluding explanations and some suggestions for further research is contained in the final section.

\section{Method and Methodology}

A lot of probability distribution functions have been proposed in recent past, but in present study Normal, Log-normal, Weibull, Gamma, Cauchy, Logistic, and Gumbel (Gumbel, 1960) are used to describe the characteristics of earthquake magnitude levels in Bangladesh. Parameters defining each distribution function are calculated using maximum likelihood method. The probability density function (PDF) of the Weibull distribution with two parameters is given by Weibull (1951). The Log-normal distribution is a probability distribution of a random variable whose logarithm is normally distributed. The probability density function (PDF) of the log-normal distribution is given by Johnson et al. (1994). Lancaster (1966) quotes from Laplace in which the latter obtains a Gamma distribution.

Cumulative Distribution Function: As indicated at the end of the introduction this study will use the widely used the Normal, Log-normal, Weibull, Gamma, Cauchy, Logistic, and Gumbel distributions to fit the earthquake magnitude (in Richter scale) in Bangladesh albeit the extensive literature experienced the use of many probability distributions in recent past. In doing so, the cumulative distribution functions of the considered probability densities are needed. They are given in Table 1 along with their parameters involve along with some remarks on them.

Table 1. Cumulative distribution function with parameters of the probability density functions in this study

\begin{tabular}{|c|c|c|c|}
\hline Distribution & Cumulative Distribution Function & Parameter & Remarks \\
\hline Normal & $F(x)=\Phi\left(\frac{x-\mu}{\sigma}\right)$ & $\begin{array}{l}\mu: \text { Location parameter } \\
\sigma: \text { Scale parameter }\end{array}$ & $\begin{array}{l}\text { Laplace integral } \\
\Phi(z)=(\sqrt{2 \pi})^{-1} \int_{0}^{z} e^{-t^{2} / 2} d t\end{array}$ \\
\hline Log-normal & $F(x)=\Phi\left[\frac{\ln (x-\gamma)-\mu}{\sigma}\right]$ & $\begin{array}{l}\gamma: \text { Loaction parameter } \\
\mu: \text { Scale parameter } \\
\sigma: \text { Shape parameter }\end{array}$ & $\begin{array}{r}\gamma=0 \text { yields two parameter } \\
\text { Lognormal distribution }\end{array}$ \\
\hline Weibull & $F(x)=1-\exp \left[-\left(\frac{x-\gamma}{\beta}\right)^{\alpha}\right]$ & $\begin{array}{l}\gamma: \text { Location parameter } \\
\beta: \text { Scale parameter } \\
\alpha: \text { Shape parameter }\end{array}$ & $\begin{array}{l}\gamma=0 \text { yields the two-parameter } \\
\text { Weibull distribution }\end{array}$ \\
\hline Gamma & $F(x)=\frac{\Gamma_{((x-\gamma) / \beta)}(\alpha)}{\Gamma(\alpha)}$ & $\begin{array}{l}\gamma: \text { Location parameter } \\
\beta: \text { Scale parameter } \\
\alpha: \text { Shape parameter }\end{array}$ & $\begin{array}{c}\Gamma_{z}: \text { Incomplete gamma function } \\
\gamma=0 \text { yields two parameter } \\
\text { Gamma distribution }\end{array}$ \\
\hline Cauchy & $F(x)=\frac{1}{2}+\frac{1}{\pi} \tan ^{-1}\left(\frac{x-\mu}{\sigma}\right)$ & $\begin{array}{l}\mu: \text { Location parameter } \\
\sigma: \text { Scale parameter }\end{array}$ & \\
\hline Logistic & $F(x)=\left[1+\exp \left(\frac{x-\mu}{\sigma}\right)\right]^{-1}$ & $\begin{array}{l}\mu: \text { Location parameter } \\
\sigma: \text { Scale parameter }\end{array}$ & \\
\hline Gumbel & $F(x)=\exp \left[-\exp \left\{-\left(\frac{x-\mu}{\sigma}\right.\right.\right.$ & $\begin{array}{l}\mu: \text { Location parameter } \\
\sigma: \text { Scale parameter }\end{array}$ & \\
\hline
\end{tabular}

Goodness-of-fit Tests: To evaluate the goodness-of-fit of the probability distributions functions fitted for the earthquake magnitude of Bangladesh will be tested by the Anderson-Darling (AD), Cramer-von Mise (CvM) and 
Kolmogorov-Smirnov (KS) test statistic and will be judged by the value of the log-likelihood, Akaike information criterion (AIC), and Bayesian information criterion (BIC) (Table 2).

Table 2. Goodness of fit statistic

\begin{tabular}{lll}
\hline Statistic & General Formula & Computational Formula \\
\hline Anderson-Darling (AD) & $n \int_{-\infty}^{\infty} \frac{\left[F_{n}(x)-F(x)\right]^{2}}{F(x)[1-F(x)]} d x$ & $-n-\frac{1}{n} \sum_{i=1}^{n}(2 i-1) \log \left[F_{i}\left(1-F_{n+1-i}\right)\right]$ \\
Cramer-von Mise (CvM) & $n \int_{-\infty}^{\infty}\left[F_{n}(x)-F(x)\right]^{2} d x$ & $\frac{1}{12 n}+\sum_{i=1}^{n}\left(F_{i}-\frac{2 i-1}{n}\right)^{2}$ \\
& $\max \left(D^{+}, D^{-}\right)$with & $\triangleq F\left(x_{i}\right)$ \\
Kolmogorov-Smirnov (KS) & $\sup \left|F_{n}(x)-F(x)\right|$ & $D^{+}=\max _{i=1, \ldots, n}\left(\frac{i}{n}-F_{i}\right)$ and $D^{-}=\max _{i=1, \ldots, n}\left(F_{i}-\frac{i-1}{n}\right)$ \\
\hline
\end{tabular}

\section{Results and Discussions}

The earthquake magnitudes (in Richter scale) in Bangladesh are made use of fitting the probability distributions the normal with parameters mean and standard deviation, Weibull with parameters shape and scale, Gamma with parameters shape and rate, log-normal with parameters log mean and log standard deviation, exponential with parameter rate, Gumbel with parameters location and scale, and Cauchy and logistic with parameters location and scale, while the parameters of the distributions are estimated through iterative maximum likelihood method and the findings are presented in Figure 2 to Figure 3 to see the fitting in terms of cumulative distribution functions plots, P-P plots, and probability density plots with the histogram.

Table 3. Summary Statistics of earthquake magnitude (in Richter scale) in Bangladesh for the period of July 08, 1918 to December 08, 2016

\begin{tabular}{ll}
\hline & Summary Statistics \\
\hline Mean & 4.65 \\
Standard Error & 0.04 \\
Median & 4.50 \\
Mode & 4.50 \\
Standard Deviation & 0.88 \\
Sample Variance & 0.77 \\
Kurtosis & 2.30 \\
Skewness & 1.01 \\
Range & 5.90 \\
Minimum & 2.80 \\
Maximum & 8.70 \\
Count & 449 \\
Confidence Level $(95.0 \%)$ & 0.08 \\
\hline
\end{tabular}

The earthquake magnitudes levels (in Richter scale) were recorded by the Bangladesh Meteorological Department (BMD) and the required data for this research work are collected the BMD. The earthquake magnitudes levels (in Richter scale) for the period of July 08, 1918 to December 08, 2016 are taken into consideration to fit the probability distribution. In order to have a better understanding of the earthquake magnitudes levels data, before entering into the desired analysis, the summary statistics for the data may be explored first (Table 3). The empirical distributional pattern of the earthquake magnitudes are explained by Box-and-Whisker plot and histogram (Figure 1). The average of earthquake magnitude levels and the average distance between earthquake magnitude levels are 4.65 and 0.88 respectively (Table 3 ), and the Box-and-Whisker plot shows that the shape of the distribution is 
positively skewed (Figure 1). In the study period, the maximum and minimum earthquake magnitude levels are 2.80 and 8.70 respectively, and the median and mode earthquake magnitude is 4.50 (Table 3 ).

(a) Box-and-Whisker plot for Earthquake Magnitude

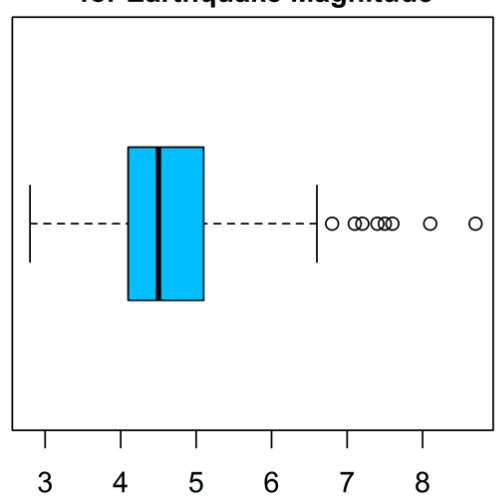

Magnitude (in Richter's Scale) (b) Histogram for Earthquake Magnitude

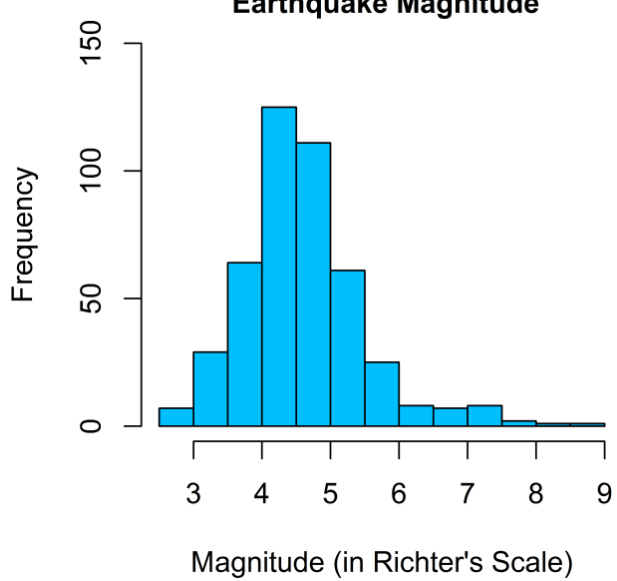

Figure 1. Schematic plots (a) Box-and-Whisker and (b) Histogram for Earthquake magnitude (in Richter scale) in Bangladesh

Table 4. Estimated parameters of different PDFs for Earthquake magnitude (in Richter scale) in Bangladesh

\begin{tabular}{llll}
\hline Distribution & Parameter & Estimate & Standard Error \\
\hline \multirow{2}{*}{ Normal } & Mean & 4.6496659 & 0.041279200 \\
& Standard Deviation & 0.8746905 & 0.029188630 \\
Log-normal & Log Mean & 1.5200716 & 0.008563375 \\
& Log Standard Deviation & 0.1814547 & 0.006054393 \\
Weibull & Shape & 5.0411960 & 0.160670100 \\
& Scale & 5.0175480 & 0.049910280 \\
Gamma & Shape & 30.060220 & 1.995213800 \\
& Rate & 6.4649900 & 0.432699700 \\
Cauchy & Location & 4.5506112 & 0.030863130 \\
& Scale & 0.4302302 & 0.026991940 \\
Logistic & Location & 4.5926646 & 0.037491130 \\
& Scale & 0.4631443 & 0.018464550 \\
Gumbel & Location & 4.2481219 & 0.036644290 \\
& Scale & 0.7353262 & 0.025918310 \\
\hline
\end{tabular}

The Figure 1.b and the value of Kurtosis in Table 3 explain the pattern of distribution of earthquake magnitude levels (in Richter scale) is leptokurtic. The standard error and the $95 \%$ confidence levels of mean earthquake magnitude levels are 0.04 and 0.08 respectively. The earthquakes take place within the study period with the interval 5.90 which contains all the earthquake magnitude levels. The Box-and-Whisker plot represents very few outliers out of 449 earthquakes magnitude levels in Richter scale which are greater than 6 (Figure 1.a).

The parameters of the distributions - Normal, Log-normal, Weibull, Gamma, Cauchy, Logistic, and Gumbel for the earthquake magnitude levels are estimated through iterative maximum likelihood method and the estimated parameters of the distributions are presented in Table 4. The functional form of log-normal distribution with in shape is $f(x)=\frac{1}{x} \frac{1}{(0.1814547) \sqrt{2 \pi}} \exp \left(-\frac{(\ln x-1.5200716)^{2}}{2(0.1814547)^{2}}\right) I_{(0, \infty)}(x)$. The standard error of the parameters 
of log-normal distribution - Log Mean and Log Standard Deviation are 0.008563375, 0.006054393 respectively (Table 4). The shape and scale parameters with the standard error of Weibull distribution are 5.0411960 $(0.160670100)$ and $5.0175480(0.049910280)$, and the shape and rate parameters with the standard error of Gamma distribution are 30.060220 (1.995213800) and 6.4649900 (0.432699700). The location and scale parameter with standard error of Cauchy distribution, Logistic distribution, and Gumbel distribution are $4.5506112(0.030863130)$ and $0.4302302 \quad(0.026991940), 4.5926646 \quad(0.037491130)$ and 0.4631443 $(0.018464550)$, and $4.2481219(0.036644290)$ and $0.7353262(0.025918310)$ respectively (Table 4$)$. The estimated parameter mean and standard deviation of the normal distribution with standard error are 4.6496659 $(0.041279200)$ and $0.8746905(0.029188630)$ correspondingly.

Table 5. Goodness of Fit Statistics and goodness of fit criteria for Earthquake magnitude (in Richter scale) in Bangladesh

\begin{tabular}{|c|c|c|c|c|c|c|}
\hline \multirow[b]{2}{*}{ Distribution } & \multicolumn{3}{|c|}{ Goodness-of-fit statistics } & \multicolumn{3}{|c|}{ Goodness-of-fit criteria } \\
\hline & $\begin{array}{l}\text { Kolmogorov } \\
\text {-Smirnov } \\
\text { Statistic }\end{array}$ & $\begin{array}{l}\text { Cramer-von } \\
\text { Mises } \\
\text { Statistic }\end{array}$ & $\begin{array}{l}\text { Anderson } \\
\text {-Darling } \\
\text { Statistic }\end{array}$ & $\begin{array}{l}\text { Log } \\
\text {-likelihood }\end{array}$ & $\begin{array}{l}\text { Akaike's Information } \\
\text { Criterion }\end{array}$ & $\begin{array}{l}\text { Bayesian Information } \\
\text { Criterion }\end{array}$ \\
\hline Normal & 0.09546651 & 0.82045974 & 5.24747992 & -576.9889 & 1157.978 & 1166.192 \\
\hline Log-normal & 0.07084472 & 0.37513413 & 2.22393502 & -553.2851 & 1110.570 & 1118.784 \\
\hline Weibull & 0.12270620 & 2.08701580 & 13.2806041 & -615.4189 & 1234.838 & 1243.052 \\
\hline Gamma & 0.07039661 & 0.44335977 & 2.75169908 & -558.0622 & 1120.124 & 1128.338 \\
\hline Cauchy & 0.07811663 & 0.41977498 & 4.48788738 & -599.5667 & 1203.133 & 1211.348 \\
\hline Logistic & 0.05506056 & 0.19940353 & 1.90841191 & -558.6588 & 1121.318 & 1129.532 \\
\hline Gumbel & 0.09814120 & 0.63268540 & 3.43249920 & -556.1773 & 1116.355 & 1124.569 \\
\hline
\end{tabular}

The Kolmogorov-Smirnov, Cramer-von Mises, and Anderson-Darling goodness of fit statistics are computed for the fitted probability distribution for the earthquake magnitude levels in Bangladesh and the best-fitted probability distributions are found for the minimum value of Akaike information criterion and Bayesian information criterion as the goodness of fit criteria. The value of Anderson-Darling Statistic for the Logistic distribution, Log-normal distribution, Gamma distribution, Gumbel distribution, Cauchy distribution, Normal distribution, and Weibull distribution are 1.90841191, 2.22393502, 2.75169908, 3.4324992, 4.48788738, 5.24747992, and 13.2806041 respectively (Table 5).

\section{(a) Emperical and Theoritical CDFs}

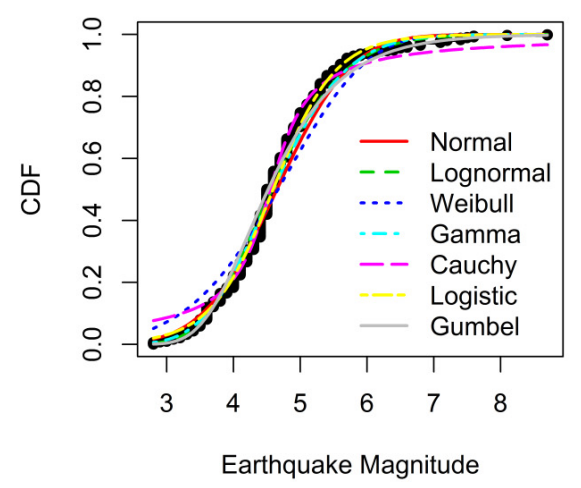

(b) P-P Plot

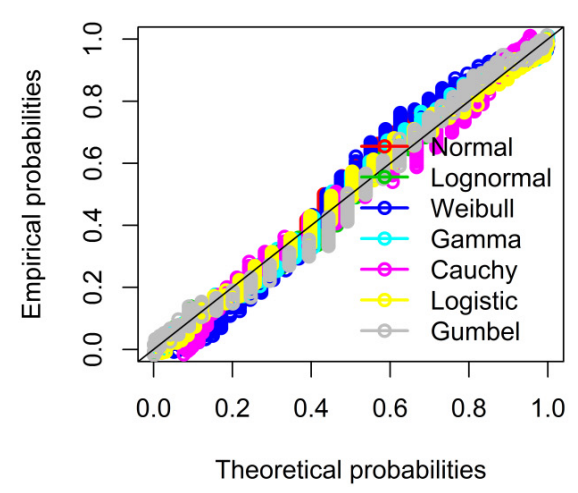

Figure 2. Schematic plots (a) Cumulative Density Functions and (b) P-P Plots for different distributions of Earthquake magnitude levels (in Richter scale) in Bangladesh 
The values of the goodness of fit statistic - Cramer-von Mises statistic for the Logistic distribution, Log-normal distribution, Cauchy distribution, Gamma distribution, Gumbel distribution, Normal distribution, and Weibull distribution are $0.19940353,0.37513413,0.41977498,0.44335977,0.6326854,0.82045974$, and 2.0870158 respectively. The distribution with the value of the Kolmogorov Smirnov goodness of fit statistic are Logistic distribution (0.05506056), Gamma distribution (0.07039661), Log-normal distribution (0.07084472), Cauchy distribution (0.07811663), Normal distribution (0.09546651), Gumbel distribution (0.0981412), Weibull distribution (0.1227062).

\section{Histogram and Theoritical Densities}

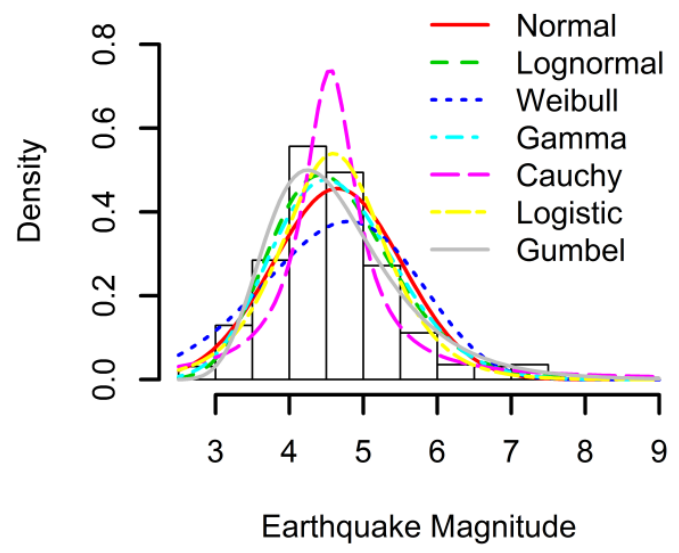

Figure 3. Density functions of different distribution for earthquake magnitude (in Richter scale) in Bangladesh

The Log-likelihood values for the fitted Weibull distribution, Cauchy distribution, Normal distribution, Logistic distribution, Gamma distribution, Gumbel distribution, Log-normal distribution are lying between -615.4 to -553.2 and maximum Log-likelihood obtains for the log-normal distribution. The Akaike's Information Criterion (AIC) values for the fitted models Log-normal, Gumbel, Gamma, Logistic, Normal, Cauchy, and Weibull are 1110.570, $1116.355,1120.124,1121.318,1157.978,1203.133$, and 1234.838 respectively (Table 5 ). The minimum AIC value gets hold of Log-normal distribution. The fitted distributions with the Goodness-of-fit criteria - Bayesian Information Criterions are Weibull (1243.052), Cauchy (1211.348), Normal (1166.192), Logistic (1129.532), Gamma (1128.338), Gumbel (1124.569), and Log-normal (1118.784); and the minimum BIC value attains for the Log-normal distribution. The findings can be used for the future plan, welfare to mankind of the country. Therefore, getting the idea about the distribution of earthquake may help a lot in the policy-making decision in different sectors.

Table 6. Probability based on Log-normal distribution for the different range of the earthquake magnitude levels (in Richter scale) in Bangladesh

\begin{tabular}{ll}
\hline $\begin{array}{l}\text { Earthquake Magnitude levels } \\
\text { (in Richter scale) }\end{array}$ & Probability based on Log-normal distribution \\
\hline$=<2.8$ & 0.003437 \\
2.8 to 3.0 & 0.006662 \\
3.0 to 4.0 & 0.220387 \\
4.0 to 5.0 & 0.458331 \\
5.0 to 6.0 & 0.244023 \\
6.0 to 7.0 & 0.057693 \\
7.0 to 8.0 & 0.008442 \\
8.0 to 8.7 & 0.000829 \\
$>=8.7$ & 0.000196 \\
\hline
\end{tabular}


The parameters of the fitted probability distributions are estimated by the maximum likelihood estimation method. The Kolmogorov-Smirnov, Cramer-von Mises, and Anderson-Darling goodness of fit statistics are computed for the fitted probability distribution for the earthquake magnitude levels (in Richter scale) in Bangladesh. The quality-of-fit of the probability distributions are tested by using the goodness-of-fit statistic the Anderson-Darling (AD), Cramer-von Mise (CvM), Kolmogorov-Smirnov (KS) and the best-fitted probability distributions for the earthquake data of Bangladesh are found to be based on the goodness-of-fit criterion for the maximum value of the log likelihood, the minimum value of Akaike information criterion (AIC), and the minimum value of Bayesian Information Criterion (BIC). The cumulative density functions and P-P plots for different distributions of earthquake magnitude levels (in Richter scale) in Bangladesh are presented in Figure 2 to acquire and recognize the best fitted distribution for the earthquake magnitude levels. The fitted density functions of different distribution for earthquake magnitude levels (in Richter scale) in Bangladesh are plotted with histogram (Figure 3). The probabilities based on the best-fitted distribution for different intervals of earthquake magnitude levels evaluate and demonstrate in Table 6 . There are 67 hundredth percent chance to occur the earthquake magnitude within 2.8 to 3,22 percent chance to occur the earthquake magnitude 3 to 4,24 percent chance to the earthquake magnitude 4 to 5 , approximately 6 percent chance to the earthquake magnitude 5 to 6 and 94 hundredth percent chance to occur the earthquake magnitude greater than 6 (Table 6).

\section{Summary and Conclusion}

The earthquake magnitudes (in Richter scale) in the study period (July 08, 1918 to December 08, 2016) lie down within greater than two to less than nine; and mean value, median value, and mode value of earthquake magnitude levels are greater or equal to four and half. The average distance between the earthquake magnitude levels is less than one. The shape of the distribution of earthquake magnitude levels is leptokurtic and positively skewed with few outliers. The maximum likelihood method is applied to estimate the parameters of the different expected distributions- Normal, Log-normal, Weibull, Gamma, Cauchy, Logistic, and Gumbel; and the parameters of these distributions are estimated with minimum standard errors. The different expected probability distributions of the earthquake magnitude levels in Bangladesh are fitted and it is found that for the data, the Log-normal distribution fits well based on graphical assessment and goodness-of-fit criterion. This research work identified that the Log-normal distribution provided the best fit for the observed earthquake magnitude levels among the probability distribution considered in this paper.

\section{Acknowledgement}

Authors are greatly thankful to the Bangladesh meteorological department for providing the data necessary for this study and authors are thankful to Jahangirnagar University to support the fund. Authors are also thankful to the editor and the anonymous reviewers for their valuable suggestions that improved the quality of this manuscript.

\section{Conflict of interests}

The authors declare that there is no conflict of interests regarding the publication of this paper.

\section{References}

Abyani, M., Asgarian, B., \& Zarrin, M. (2019). Sample geometric mean versus sample median in closed form framework of seismic reliability evaluation: A case study comparison. Earthq Eng \& Eng Vib, 18(1), 187-201.

Al Zaman, M. D. A., \& Monira, N. J. (2017). A Study of Earthquakes in Bangladesh and the Data Analysis of the Earthquakes that were generated In Bangladesh and Its' Very Close Regions for the Last Forty Years (1976-2016). J Geol Geophys, 6(4), 1-5.

Ansary, M. A. (2002). Earthquake damage scenario for Sylhet, Bangladesh, In Seventh U.S. National Conference on Earthquake Engineering. Boston, Massachusetts, USA.

Ara, S. (2013). Analyzing population distribution and its effect on earthquake loss estimation in Sylhet, Bangladesh. Applied Earth Science Faculty of Geoinformation Science and Earth Observation, University of Twente, Enschede, the Netherlands.

Ara, S. (2014). Impact of Temporal Population Distribution on Earthquake Loss Estimation: A Case Study on Sylhet, Bangladesh. Int J Disaster Risk Sci, 5, 296-312.

Bari, M. S., \& Das, T. (2013). A Comparative Study on Seismic Analysis of Bangladesh National Building Code (BNBC) with Other Building Codes. J. Inst. Eng. India Ser. A, 94(3), 131-137.

Bernardo, J. M., \& Smith, A. F. M. (1994). Bayesian Theory. Wiley, Chichester. 
Bhattacharya, P., \& Bhattacharjee, R. (2009). A Study on Weibull Distribution for Estimating the Parameters. Wind Engineering, 33(5), 469-476.

Charpentier, A., \& Durand, M. (2015). Modeling earthquake dynamics. J Seismol, 19(3), 721-739.

Delignette-Muller, M. L., \& Dutang, C. (2015). Fitdistrplus: An R Package for Fitting Distributions. Journal of Statistical Software, 64(4), 1-34.

Dhanya, J., \& Raghukanth, S. T. G. (2019). A non-Gaussian random field model for earthquake slip. J Seismol, 23(4), 889-912.

Ferraes, S. G. (2003. The conditional probability of earthquake occurrence and the next large earthquake in Tokyo, Japan. J Seismol, 7(2), 145-153.

Gumbel, E. J. (1960). Multivariate Extremal Distributions. Bull. Inst. Internat. de Statistique, 37, 471-475.

Johnson, L., Norman, K. S., \& Balakrishnan, N. (1994). Continuous univariate distributions. Wiley Series in Probability and Mathematical Statistics: Applied Probability and Statistics (Vol. 1, 2nd ed.). New York: John Wiley \& Sons.

Kislov, K. V., \& Gravirov, V. V. (2018). Deep Artificial Neural Networks as a Tool for the Analysis of Seismic Data. Seism. Instr., 54(8), 8-16.

Lancaster, H. O. (1966). Forerunners of the Pearson Chi-square. Australian Journal of Statistics, 8, 117-126.

Lillo, C., Leiva, V., Nicolis, O., \& Aykroyd, R. G. (2018). L-moments of the Birnbaum-Saunders distribution and its extreme value version: Estimation, goodness of fit and application to earthquake data. Journal of Applied Statistics, 45(2), 187-209.

Matthews, M. V., Ellsworth, W. L., \& Reasenberg, P. A. (2002). A Brownian model for recurrent earthquakes. Bull. seism. Soc. Am., 92, 2233-2250.

Mazzotti, S., \& Adams, J. (2005). Rates and uncertainties on seismic moment and deformation in eastern Canada. J. Geophys. Res., 110, B09301.

Mohammadi, H., \& Bayrak, Y. (2016). An evaluation of earthquake hazard parameters in the Iranian Plateau based on the Gumbel III distribution. J Seismol, 20(2), 615-628.

Nichols, K., Trevino, E., Ikeda, N., Philo, D., Garcia, A., \& Bowman, D. (2018). Interdependency amongst earthquake magnitudes in Southern California. Journal of Applied Statistics, 45(4), 763-774.

Parker, R., Hancox, G., Petley, D., Massey, C., Densmore, A., \& Rosser, N. (2015). Spatial distributions of earthquake-induced landslides and hill slope preconditioning in the northwest South Island, New Zealand. Earth Surf Dyn, 3, 501-525.

Pasari, S. (2019). Inverse Gaussian versus lognormal distribution in earthquake forecasting: Keys and clues. $J$ Seismol, 23(3), 537-559.

Patel, J. K., Kapadia, C. H., \& Owen, D. B. (1976). Handbook of Statistical Distributions. Marcel Dekker, New York.

Rahman, F., Islam, J., Mohammad, M., Chakravorti, B. K., \& Sarkar, P. (2018). Study of the Earthquake Probabilities in the Five Major Faults in and around Bangladesh: A Statistical Analysis. J Earth Sci Clim Change, 9(1(438)), 1-5.

Rahman, M. H., Matin, M. A., \& Salma, U. (2018). Analysis of precipitation data in Bangladesh through hierarchical clustering and multidimensional scaling. Theor Appl Climatol, 134, 689-705.

Rahman, S. M., Rahman, M. H., Faruk, M. O., \& Sultan-Ul-Islam, M. (2018). Seismic Status in Bangladesh. Vietnam Journal of Earth Sciences, 40(2), 178-192.

Roback, K., Clark, M. K., West, A. J., Zekkos, D., Li, G., Gallen, S. F., Chamlagain, D., \& Godt, J. W. (2018). The size, distribution, and mobility of landslides caused by the $2015 \mathrm{Mw} 7.8$ Gorkha earthquake, Nepal. Geomorphology, 301, 121-138.

Roy, S. (2014). Probabilistic Prediction for Earthquake in Bangladesh: Just How Big Does the Earthquake Have to Be Next Years? Open Journal of Earthquake Research, 3(2), 108-114.

Sornette, D. (2000). Critical Phenomena in Natural Sciences. Springer, Berlin, Heidelberg, New York.

Tanyaş, H. J., van Westen, C., Persello, C., \& Alvioli, M. (2019). Rapid prediction of the magnitude scale of landslide events triggered by an earthquake. Landslides, 16, 661-676. 
Tian, Y., Xu, C., Hong, H., Zhou, Q., \& Wang, D. (2019). Mapping earthquake-triggered landslide susceptibility by use of artificial neural network (ANN) models: An example of the 2013 Minxian (China) Mw 5.9 event, Geomatics. Natural Hazards and Risk, 10(1), 1-25

Weibull, W. (1951). A statistical distribution function of wide applicability. J. Appl. Mech. Trans., ASME, 18(3), 293-297.

Yakovlev, G., Turcotte, D. L., Rundle, J. B., \& Rundle, P. B. (2006). Simulation-based distributions of earthquake recurrence times on the San Andreas fault system. Bulletin of the Seismological Society of America, 96, 1995-2007.

Zöller, G., Ben-Zion, Y., Holschneider, M., \& Hainzl, S. (2007). Estimating recurrence times and seismic hazard of large earthquakes on an individual fault. Geophysical Journal International, 170(3), 1300-1310.

\section{Copyrights}

Copyright for this article is retained by the author(s), with first publication rights granted to the journal.

This is an open-access article distributed under the terms and conditions of the Creative Commons Attribution license (http://creativecommons.org/licenses/by/4.0/). 